\title{
Online Edge Coloring of Paths and Trees with a Fixed Number of Colors
}

\author{
Lene M. Favrholdt • Jesper W. Mikkelsen
}

the date of receipt and acceptance should be inserted later

\begin{abstract}
We study a version of online edge coloring, where the goal is to color as many edges as possible using only a given number, $k$, of available colors. All of our results are with regard to competitive analysis. Previous attempts to identify optimal algorithms for this problem have failed, even for bipartite graphs. Thus, in this paper, we analyze even more restricted graph classes, paths and trees. For paths, we consider $k=2$, and for trees, we consider any $k \geq 2$.

We prove that a natural greedy algorithm called FIRST-FIT is optimal among deterministic algorithms, on paths as well as trees. For paths, we give a randomized algorithm, which is optimal and better than the best possible deterministic algorithm. For trees, we prove that to obtain a better competitive ratio than FIRST-FIT, the algorithm would have to be both randomized and unfair (i.e., reject edges that could have been colored), and even such algorithms cannot be much better than FIRST-FiT.
\end{abstract}

\footnotetext{
A preliminary version of this paper appeared in 12th Workshop on Approximation and Online Algorithms (WAOA 2014), LNCS 8952: 181-192, 2014.

This work was partially supported by the Villum Foundation and the Danish Council for Independent Research, Natural Sciences.

Lene M. Favrholdt (凶)

Department of Mathematics and Computer Science, University of Southern Denmark, Campusvej 55, 5230 Odense M, Denmark

Tel.: +4565502341

E-mail: lenem@imada.sdu.dk

Jesper W. Mikkelsen

Department of Mathematics and Computer Science, University of Southern Denmark

Campusvej 55, 5230 Odense M, Denmark

E-mail: jesperwm@imada.sdu.dk
} 


\section{Introduction}

In the classical edge coloring problem, the edges of a graph must be colored using as few colors as possible, under the constraint that no two adjacent edges receive the same color. There is a natural dual version of the problem where a fixed number, $k$, of colors is given and the goal is to color as many edges as possible, using at most $k$ colors. Sometimes the classical problem is called the minimization version and the dual problem is called the maximization version of the problem.

In this paper, we study the following online version of the maximization problem 9]: The edges of the graph arrive one by one (in any order), each specified by its endpoints. Immediately upon receiving an edge, the algorithm must either color the edge with one of the $k$ colors or reject the edge. The decision of which of the $k$ colors to use or to reject the edge is irrevocable. We call this problem EdGE- $k$-Coloring. For any class, Class, of graphs, we let Edge- $k$-Coloring(Class) denote the problem of EdGE- $k$-Coloring restricted to graphs of class Class. For instance, EdGE-2-Coloring(PATH) is the online problem of properly coloring as many edges as possible in a path using only two colors.

Quality measure. We measure the quality of an online algorithm, A, for EDGE$k$-COLORING using the standard notion of competitive ratio [12,16]. The competitive ratio compares the performance of A to that of an optimal offline algorithm, Opт. We denote by $\mathrm{A}(\sigma)$ the number of edges colored by $\mathrm{A}$ when given a sequence, $\sigma$, of edges. Similarly, $\operatorname{OpT}(\sigma)$ is the number of edges in $\sigma$ colored by Opt. The algorithm A is said to be $C$-competitive if there exists a constant $b$ such that $\mathrm{A}(\sigma) \geq C \cdot \operatorname{OPT}(\sigma)-b$ for any input sequence $\sigma$. The competitive ratio, $C_{\mathrm{A}}(k)$, of $\mathrm{A}$ is the supremum over all $C$ for which $\mathrm{A}$ is $C$-competitive. The competitive ratio of A for Edge- $k$-Coloring(Class) is denoted by $C_{\mathrm{A}}^{\text {CLASS }}(k)$.

Note that by this definition, $0 \leq C_{\mathrm{A}}(k) \leq 1$. In particular, upper bounds on the competitive ratio are negative results and lower bounds are positive results.

If the inequality above holds even when $b=0$, we say that $\mathrm{A}$ is strictly $C$ competitive. This gives rise to the notion of strict competitive ratio. The results in this paper are strongest possible in the sense that all positive results hold for the strict competitive ratio and all negative results hold for the competitive ratio.

For randomized algorithms, a similar definition of competitive ratio is used but $\mathrm{A}(\sigma)$ is replaced by the expected value $\mathbb{E}[\mathrm{A}(\sigma)]$.

Notation and terminology. We label the $k$ colors $1,2, \ldots, k$. For $1 \leq i \leq j \leq k$, define $\mathcal{C}_{i, j}=\{i, i+1, \ldots, j\}$. At any fixed point in the processing of the input sequence, we denote by $\mathcal{C}_{v}$ the set of colors used at edges incident to the vertex $v$. A color $i \in \mathcal{C}_{1, k}$ is said to be available at $v$ if $i \notin \mathcal{C}_{v}$. Two colorings of a graph 
are said to be equivalent if one can be obtained from the other by renaming the colors.

If $v$ is a vertex in the input graph, we denote by $d(v)$ the number of edges incident to $v$. An isolated edge $e=(v, u)$ is an edge such that $d(v)=d(u)=1$ at the time when $e$ is revealed. For any $m$, we let $\left\langle e_{1}, e_{2}, \ldots, e_{m}\right\rangle$ denote a path with $m$ edges labeled such that, for $2 \leq i \leq m-1, e_{i}$ is adjacent to $e_{i-1}$ and $e_{i+1}$. A star with $m$ edges is the complete bipartite graph $K_{1, m}$.

Algorithms. An algorithm is called fair if it never rejects an edge unless all of the $k$ colors have already been used on adjacent edges. In [9], the following two fair deterministic algorithms were studied:

FIRST-Fit (FF) uses the lowest available color for each edge. It can be viewed as the natural greedy strategy.

NeXT-Fit (NF) remembers the last used color $c_{\text {last }}$. For each edge, it uses the first available color in the ordered sequence $\left\langle c_{\text {last }}+1, \ldots, k, 1, \ldots, c_{\text {last }}\right\rangle$. For the very first edge, it uses the color 1.

For EdGe-2-Coloring(PATH), we introduce a new family of randomized algorithms: For $\frac{1}{2} \leq p \leq 1, \mathrm{RAND}_{p}$ is defined as follows. Whenever an isolated edge is revealed, $\operatorname{RAND}_{p}$ uses the color 1 with probability $p$ and the color 2 with probability $1-p$. All non-isolated edges are colored (with the only remaining color) if possible. Note that RAND 1 is identical to FIRST-FIT.

Previous results. In [9] it is shown that any fair algorithm for EDGE- $k$-COLORING has a competitive ratio of at least $2 \sqrt{3}-3 \approx 0.46$, and at most $\frac{1}{2}$ if it is deterministic. The lower bound is tight in the sense that NEXT-FIT has a competitive ratio of exactly $2 \sqrt{3}-3$. The competitive ratio of FIRST-FIT is at most $\frac{2}{9}(\sqrt{10}-1) \approx 0.48$. It remains an open problem whether there is an algorithm with a competitive ratio better than $2 \sqrt{3}-3$. It is also shown that no algorithm (even when allowing randomization) has a competitive ratio better than $\frac{4}{7} \approx 0.57$.

The problem EDGE- $k$-Coloring $(k$-Colorable $)$ is also studied in $[9]$. When the input graph is $k$-colorable, any fair algorithm is shown to have a competitive ratio of at least $\frac{1}{2}$. Again, the lower bound is tight because NEXT-FIT has a competitive ratio of $\frac{1}{2}$. The competitive ratio of FIRST-FIT is shown to be $\frac{k}{2 k-1}$. An upper bound of $\frac{2}{3}$ is given for deterministic algorithms in this case.

We remark that all of the negative results mentioned above hold even if the input graph is bipartite. Thus, contrary to offline edge coloring, the online EDGE- $k$-COLORING problem does not appear to be significantly easier when restricted to bipartite graphs.

It is well known that for $k=1$ (i.e., for the matching problem), the greedy algorithm is an optimal deterministic algorithm with a competitive ratio of $\frac{1}{2}$.

The relative worst order ratio [4,5] of both the maximization and minimization version of online edge coloring is studied in $[8]$. For the maximization 
version, it is shown that FIRST-FIT and NEXT-FIT are not (strictly) comparable. This is true even when the input is restricted to bipartite graphs. For the minimization version, FIRST-FIT is proven better than NEXT-FIT.

The minimization version of online edge coloring is studied in [2. If an online algorithm never introduces a new color unless forced to do so, it will never use more than $2 \Delta-1$ different colors on graphs of maximum degree $\Delta$. It is shown in 2] that no (randomized) online algorithm can do better than this, even if the input graph is restricted to being a forest. On any graph, an optimal offline algorithm uses at most $\Delta+1$ colors, and on trees, $\Delta$ colors suffice. Hence, any algorithm that introduces a new color only when necessary, has a competitive ratio of 2 , and this is optimal.

The problem of online vertex coloring has received much attention in the minimization version (see [13] for a survey). For interval graphs, it has also been studied in the maximization version: It follows from a result in [6] that no deterministic fair algorithm can have a competitive ratio strictly greater than 0 , even on interval graphs. In that paper it is also shown that, on $k$-colorable interval graphs, any fair algorithm has a competitive ratio of at least $\frac{1}{2}$. In 1 , it is shown that for deterministic algorithms, this lower bound is tight, i.e., any deterministic fair algorithm has a competitive ratio of exactly $\frac{1}{2}$ on $k$ colorable interval graphs. Since edge coloring is equivalent to vertex coloring of line graphs, our results and those of 9 and 8 can also be seen as results on vertex coloring of (subclasses of) line graphs. In particular, edge coloring a path of $m$ edges is equivalent to vertex coloring a path of $m$ vertices.

A study of approximation algorithms for the offline maximization version of edge coloring for multigraphs was initiated in [10]. This line of work has been continued in $7,11,14,15$, for both simple graphs and multigraphs.

Our contribution. For EdGE-2-Coloring(PATH), we give a $\frac{4}{5}$-competitive randomized algorithm and prove that this is optimal. We also show that no deterministic algorithm can be better than $\frac{2}{3}$-competitive and observe that this upper bound is tight, since FIRST-FIT is $\frac{2}{3}$-competitive. Finally, NEXT-FIT turns out to be a worst possible fair algorithm with a competitive ratio of $\frac{1}{2}$.

For Edge- $k$-Coloring(TREE) where $k \geq 2$, we prove that FIRST-FIT is $\frac{k-1}{k}$-competitive and that no deterministic or fair algorithm can be better than this. Thus, an algorithm would have to be both randomized and unfair to achieve a better competitive ratio than FIRST-FIT. However, we show that even such algorithms cannot be better than $\frac{k}{k+1}$-competitive. We also show that any fair algorithm is $\frac{2 \sqrt{k}-2}{2 \sqrt{k}-1}$-competitive and that the competitive ratio of NEXT-FIT is no better than this if $k$ is a square number. This implies that the competitive ratio of any fair algorithm goes to 1 as $k$ goes to infinity.

PATH and TREE are the first examples of graph classes for which an optimal deterministic algorithm for EDGE- $k$-COLORING has been identified. PATH is the first graph class for which an optimal randomized algorithm has been identified. It is also the first class for which it has been proven that a randomized algorithm can be better than a best possible deterministic algorithm. 
We remark that all of our results for PATH extend to collections of paths. Similarly, all results for TREE extend to forests. This is so because our positive results are always for the strict competitive ratio, and because our algorithms will color a single path in a collection of paths exactly as if only the edges of that path had been revealed (similarly for trees).

\section{A Charging Technique for Proving Positive Results}

We will now describe a simple charging technique for proving lower bounds on the competitive ratio. The technique was first used for deterministic algorithms in 9]. For some $C, 0 \leq C \leq 1$, our goal is to prove that a given (possibly randomized) algorithm $\mathrm{A}$ is $C$-competitive. Assume that the edges of a graph $G=(V, E)$ have been given in some order, $\sigma$, and let $E_{\text {Орт }} \subseteq E$ be the set of edges colored in some optimal solution.

The initial value $v_{\mathrm{i}}(e)$ of an edge, $e \in E$, is $v_{\mathrm{i}}(e)=\operatorname{Pr}[e$ is colored by $\mathrm{A}]$. For deterministic algorithms, $v_{\mathrm{i}}(e) \in\{0,1\}$ for all $e \in E$. Note that by linearity of expectation, we have $\mathbb{E}[\mathrm{A}(\sigma)]=\sum_{e \in E} v_{\mathrm{i}}(e)$.

The surplus $v_{+}(e)$ of an edge, $e \in E$, (with respect to $C$ ) is

$$
v_{+}(e)= \begin{cases}v_{\mathrm{i}}(e)-C, & \text { if } e \in E_{\mathrm{OPT}} \\ v_{\mathrm{i}}(e), & \text { if } e \notin E_{\mathrm{OPT}}\end{cases}
$$

We let $E_{+} \subseteq E$ and $E_{-} \subseteq E$ denote the sets of edges with positive and negative surplus, respectively. Clearly, $E_{-} \subseteq E_{\mathrm{OPT}}$. For deterministic algorithms, $E_{-}$is exactly those edges in $E_{\mathrm{OPT}}$ that are not colored by the algorithm, and $E_{+}$is the set of edges colored by the algorithm (assuming $C<1$ ). The total positive surplus $\sum_{e \in E_{+}} v_{+}(e)$ will be redistributed among the edges in $E_{-}$according to some strategy. This strategy is what needs to be defined when applying the technique.

The final value $v_{\mathrm{f}}(e)$ of an edge $e \in E_{\mathrm{Opt}}$ is the total value of $e$ after the redistribution of surplus. Since only surplus value is redistributed, $v_{\mathrm{f}}(e) \geq C$ for all $e \in E_{\text {Орт }} \backslash E_{-}$. Thus, if it can be proven that $v_{\mathrm{f}}(e) \geq C$ for all $e \in E_{-}$, then

$$
\begin{aligned}
\mathbb{E}[\mathrm{A}(\sigma)] & =\sum_{e \in E} v_{\mathrm{i}}(e) \\
& =\sum_{e \in E} v_{\mathrm{f}}(e) \\
& \geq \sum_{e \in E_{\mathrm{OPT}}} v_{\mathrm{f}}(e), \text { since } v_{\mathrm{f}}(e) \geq 0 \text { for all } e \in E \\
& \geq C \cdot \operatorname{OpT}(\sigma) .
\end{aligned}
$$

Thus, it follows that $\mathrm{A}$ is (strictly) $C$-competitive. 


\section{Coloring of Paths}

In this section, we study the EDGE- $k$-COLORING problem when the input graph is a path. This is only interesting if $k \leq 2$, since for $k \geq 3$, any fair algorithm colors all edges of any path. In this paper, we consider solely the case where $k=2$, but we remark that one can use similar techniques to obtain tight bounds on the competitive ratio when $k=1$. Also, the results for PATH can be extended to graphs of maximum degree 2 .

For EDGE-2-Coloring(PATH), our main result is a randomized algorithm with a competitive ratio of $\frac{4}{5}$ and a proof that this is optimal. Before considering randomized algorithms, we give tight lower and upper bounds on the competitive ratio of deterministic algorithms.

For 2-colorable graphs, the ratios of Propositions 1 and 2 both follow from 9 . Clearly, the positive results carry over to paths, but for $k=2$, the graphs used in [9] for the negative results are not connected. We give simple proofs that the negative results are also valid when the graph is a path.

Proposition 1 For Edge-2-Coloring(PATH), NeXt-Fit is a worst possible fair algorithm with

$$
C_{\mathrm{NF}}^{\mathrm{PATH}}(2)=\frac{1}{2} \text {. }
$$

Proof The lower bound for fair algorithms follows, since each rejected edge is adjacent to exactly two colored edges, and each colored edge is adjacent to at most two rejected edges.

For the upper bound, consider a path $\left\langle e_{1}, \ldots, e_{2 m+1}\right\rangle$ with $2 m+1$ edges. The adversary first reveals the odd-numbered edges in order of increasing indices. NEXT-FIT will alternate between the two colors. Afterwards, the adversary reveals all the even-numbered edges. These edges must all be rejected by NeXT-Fit. Thus, the competitive ratio of NEXT-FIT is at most $\frac{m+1}{2 m+1}$ which tends to $\frac{1}{2}$ as $m$ tends to infinity.

Proposition 2 For Edge-2-Coloring(PATH), First-Fit is an optimal deterministic algorithm with

$$
C_{\mathrm{FF}}^{\mathrm{PATH}}(2)=\frac{2}{3} .
$$

Proof Since a path is 2-colorable, the lower bound for FIRST-FiT follows from a result in [9] stating that the competitive ratio of FIRST-FIT is $\frac{k}{2 k-1}$ for the EdGe- $k$-COlORIng $(k$-COLORABLE) problem. It also follows from Lemma 2 below, with $p=1$.

For the upper bound, let $\mathrm{D}$ be a deterministic algorithm and let $n \in \mathbb{N}$. The adversary first gives $n$ disjoint paths of length two. Call these the initial paths. Let $F=\left\{f_{1}, \ldots, f_{n_{1}}\right\}$ be the set of those initial paths in which both edges have been colored by $\mathrm{D}$ and let $U=\left\{u_{1}, \ldots, u_{n_{2}}\right\}$ be the set of those initial paths in which at least one edge has been rejected.

In each path in $F$, both colors 1 and 2 are represented. The adversary reveals an edge connecting the edge with the color 1 in the path $f_{i}$ to the edge 
with the color 2 in the path $f_{i+1}$, for $1 \leq i<n_{1}$. These connecting edges must be rejected by $\mathrm{D}$ so the number of colored edges in this component is at most $2 n_{1}$. The adversary also reveals an edge connecting $u_{i}$ to $u_{i+1}$, for $1 \leq i<n_{2}$. Even if all of these connecting edges can be colored, the number of colored edges in this component is at most $2 n_{2}-1$.

Finally, if both $F$ and $U$ are non-empty, the adversary connects the two constructed paths by a single edge which may possibly be colored. It follows that the number of colored edges can be at most $2 n_{1}+\left(2 n_{2}-1\right)+1=2 n$. Since the total number of edges is $3 n-1$, we get an upper bound on the competitive ratio of $\frac{2 n}{3 n-1}$ which tends to $\frac{2}{3}$ as $n$ tends to infinity.

Knowing that no deterministic algorithm can be better than $\frac{2}{3}$-competitive, a natural question to ask is how good a randomized algorithm can be. To this end, we analyze the family of fair, randomized algorithms, $\mathrm{RAND}_{p}$, defined in the introduction.

Lemma 1 Let $\frac{1}{2} \leq p \leq 1$. Then,

$$
C_{\mathrm{RAND}_{p}}^{\mathrm{PATH}}(2) \leq \min \left\{p^{2}-p+1, \frac{2}{3}\left(-p^{2}+p+1\right)\right\} .
$$

Proof The adversary will reveal the edges of a path $P=\left\langle e_{1}, \ldots, e_{m}\right\rangle$ with $m$ edges. Consider the following two adversary strategies for doing so:

(i) The adversary first reveals all edges $e_{i}$ with $i \equiv 1(\bmod 3)$, followed by all edges $e_{i}$ with $i \equiv 0(\bmod 3)$. Finally, all the remaining edges are revealed.

(ii) The adversary first reveals all the odd numbered edges and thereafter all the even numbered edges.

If the adversary uses strategy (i), it chooses $m$ such that 3 divides $m-1$. Note that each edge $e_{i}$ with $i \equiv 2(\bmod 3)$ has probability $p(1-p)+(1-p) p$ of being colored. It follows that

$$
\begin{aligned}
\mathbb{E}\left[\operatorname{RAND}_{p}(P)\right] & =\left(\frac{1}{3}(m-1)+1\right)+\frac{1}{3}(m-1)+\frac{2}{3}(m-1)(1-p) p \\
& =\frac{2}{3}\left(-p^{2}+p+1\right)(m-1)+1
\end{aligned}
$$

If the adversary uses strategy (ii), it makes sure that the number, $m$, of edges in $P$ is odd. Note that each even numbered edge has probability $p^{2}+(1-p)^{2}$ of being colored. It follows that

$$
\begin{aligned}
\mathbb{E}\left[\operatorname{RAND}_{p}(P)\right] & =\left(\frac{1}{2}(m-1)+1\right)+\frac{1}{2}(m-1)\left(p^{2}+(1-p)^{2}\right) \\
& =\left(p^{2}-p+1\right)(m-1)+1 .
\end{aligned}
$$

Thus, if $\frac{2}{3}\left(-p^{2}+p+1\right) \leq p^{2}-p+1$, the adversary uses strategy (i) and otherwise it uses strategy (ii). By choosing $m$ sufficiently large, this proves the upper bound. 
Lemma 2 Let $\frac{1}{2} \leq p \leq 1$. Then,

$$
C_{\mathrm{RAND}_{p}}^{\mathrm{PATh}}(2) \geq \min \left\{p^{2}-p+1, \frac{2}{3}\left(-p^{2}+p+1\right)\right\} .
$$

Proof Let $P$ be a path and assume that the edges of $P$ are given to $\mathrm{RAND}_{p}$ in some order. Consider an edge $e$ at the time of its arrival. If two edges adjacent to $e$ have already been revealed, we say that $e$ is a critical edge. Denote by $E_{\text {crit }}$ the critical edges of $P$. Note that since $\mathrm{RAND}_{p}$ is fair, it will never reject an edge which is not critical.

We let $C=\min \left\{p^{2}-p+1, \frac{2}{3}\left(-p^{2}+p+1\right)\right\}$ and apply the charging technique described in Section 2. That is, we will define a strategy for distributing the total surplus among the edges of the path such that all edges receive a final value of at least $C$. This will imply that $\mathrm{RAND}_{p}$ is $C$-competitive. Note that all non-critical edges have an initial value of 1 and, hence, a surplus of $1-C$. Thus, $E_{-} \subseteq E_{\text {crit }}$.

Let $e$ be a non-critical edge. Consider the largest connected component $P_{e}$ induced by edges from $E \backslash E_{\text {crit }}$ containing $e$. Let $e_{\text {first }}$ be the edge in $P_{e}$ which was revealed first. We define $l(e)$ to be the length of the shortest path in $P_{e}$ containing $e$ and $e_{\text {first }}$. If $e$ is revealed as an isolated edge, then $l(e)=1$. We say that $e$ is odd if $l(e)$ is odd and that $e$ is even if $l(e)$ is even. The following fact is easily proven by induction on $l(e)$.

Fact: If $e$ is odd, the probability of e being colored with the color 1 is $p$. If $e$ is even, the probability of e being colored with the color 1 is $1-p$.

Let $e_{\text {crit }}$ be a critical edge. Denote by $e_{1}$ and $e_{\mathrm{r}}$ the two edges adjacent to $e_{\text {crit }}$. These must both be non-critical and thus must be colored by $\operatorname{RAND}_{p}$. The edge $e_{\text {crit }}$ will be colored if and only if $e_{\mathrm{l}}$ and $e_{\mathrm{r}}$ are colored with the same color. Note that the random variable denoting the color received by $e_{1}$ is independent of the random variable denoting the color received by $e_{\mathrm{r}}$. We consider two cases:

Case 1: $e_{l}$ and $e_{r}$ are both odd or both even. By the fact stated above, the probability of $e_{\text {crit }}$ being colored is $p^{2}+(1-p)^{2}$. It follows that

$$
v_{\mathrm{i}}\left(e_{\mathrm{crit}}\right)=p^{2}+(1-p)^{2}=2 p^{2}-2 p+1 \text {. }
$$

Since $e_{\mathrm{l}}$ and $e_{\mathrm{r}}$ are non-critical, they both have a surplus of at least $1-C$. We will transfer a value of $\frac{1}{2}(1-C)$ from each of them to the critical edge $e_{\text {crit }}$. Thus, the final value of $e_{\text {crit }}$ is

$$
\begin{aligned}
v_{\mathrm{f}}\left(e_{\text {crit }}\right) & \geq\left(2 p^{2}-2 p+1\right)+(1-C) \\
& =2\left(p^{2}-p+1\right)-C \\
& \geq C, \text { since } C \leq p^{2}-p+1
\end{aligned}
$$


Case 2: One of $e_{l}$ and $e_{r}$ is odd and the other is even. Without loss of generality, assume that $e_{\mathrm{l}}$ is odd and that $e_{\mathrm{r}}$ is even. By the fact stated above, the probability of $e_{\text {crit }}$ being colored is $p(1-p)+(1-p) p$. Thus,

$$
v_{\mathrm{i}}\left(e_{\text {crit }}\right)=2 p(1-p)=2\left(-p^{2}+p\right) \text {. }
$$

Since $e_{\mathrm{r}}$ is even, it must be adjacent to at least one non-critical edge $e_{\mathrm{r}}^{\prime}$. We transfer a value of $\frac{1}{2}(1-C)$ from each of $e_{\mathrm{l}}$ and $e_{\mathrm{r}}^{\prime}$ to $e_{\text {crit }}$ and a value of $1-C$ from $e_{\mathrm{r}}$ to $e_{\mathrm{crit}}$. Transferring the entire surplus of $1-C$ from $e_{\mathrm{r}}$ to $e_{\text {crit }}$ is possible, since $e_{\mathrm{r}}^{\prime}$ is non-critical and therefore $e_{\text {crit }}$ is the only critical edge adjacent to $e_{\mathrm{r}}$. Thus, the final value of $e_{\text {crit }}$ is

$$
\begin{aligned}
v_{\mathrm{f}}\left(e_{\text {crit }}\right) & =2\left(-p^{2}+p\right)+2(1-C) \\
& =2\left(-p^{2}+p+1-C\right) \\
& \geq C, \text { since } C \leq \frac{2}{3}\left(-p^{2}+p+1\right)
\end{aligned}
$$

Lemmas 1 and 2 immediately imply the following theorem.

Theorem 1 Let $\frac{1}{2} \leq p \leq 1$. Then,

$$
C_{\mathrm{RAND}_{p}}^{\mathrm{PATH}}(2)=\min \left\{p^{2}-p+1, \frac{2}{3}\left(-p^{2}+p+1\right)\right\} .
$$

Theorem 1 shows that, for $p=\varphi / \sqrt{5} \approx 0.7236, \mathrm{RAND}_{p}$ has a competitive ratio of $\frac{4}{5}$ (where $\varphi=(1+\sqrt{5}) / 2$ is the golden ratio). In practice, one might prefer that $p$ is, e.g., a dyadic rational (a rational of the form $a / 2^{b}$ for $a, b \in \mathbb{N}$ ). It follows from Theorem 1 that the competitive ratio of $\mathrm{RAND}_{p}$ can be made arbitrarily close to $\frac{4}{5}$ by choosing a dyadic rational $p$ sufficiently close to the irrational number $\varphi / \sqrt{5}$.

We will now show that $\frac{4}{5}$ is the best possible competitive ratio of any algorithm. In fact, we show that this is true even if the algorithm knows the length of the path in advance (so that only the ordering of the edges is unknown). We will use Yao's minimax principle [3,17]. Informally, this principle allows us to prove an upper bound of $c$ on the achievable randomized competitive ratio by exhibiting a probability distribution over permutations of the edges of a path and showing that no deterministic algorithm can, in expectation, color more than a fraction of $c$ of the edges of the path.

Theorem 2 If $\mathrm{R}$ is a (possibly randomized) algorithm for the problem EDGE2-Coloring(PATH), then

$$
C_{\mathrm{R}}^{\mathrm{PATH}}(2) \leq \frac{4}{5}
$$


Proof Let $M \in \mathbb{N}$ be a large even integer and consider a path $P$ consisting of $\frac{5}{2} M+1$ edges. We will define a probability distribution over all permutations of the edges of $P$ by describing a randomized adversary.

The adversary reveals the edges of $P$ as follows: First, it reveals $M+1$ isolated edges $\left\{e_{1}, \ldots, e_{M+1}\right\}$. Afterwards, the adversary picks uniformly at random a set of indices $S \subseteq\{2, \ldots, M+1\}$ such that $|S|=\frac{M}{2}$. For each index $i \in S$, the adversary reveals a single edge, $e$, connecting $e_{i}$ and $e_{i-1}$ (so that $\left\langle e_{i-1}, e, e_{i}\right\rangle$ becomes a subpath of $\left.P\right)$. Let $\bar{S}=\{1,2, \ldots, M+1\} \backslash S$. For each index $i \in \bar{S}$, the adversary reveals two edges, $e$ and $e^{\prime}$, connecting $e_{i}$ and $e_{i-1}$ (so that $\left\langle e_{i-1}, e, e^{\prime}, e_{i}\right\rangle$ becomes a subpath of $P$ ). Note that the resulting path $P$ has $M+1+\frac{M}{2}+M=\frac{5}{2} M+1$ edges.

Let $\mathrm{D}$ be any deterministic algorithm, and let $\mathbb{E}[\mathrm{D}(P)]$ denote the expected number of edges colored by $\mathrm{D}$ when the edges of $P$ are revealed as described above. We will show that $\mathbb{E}[\mathrm{D}(P)]$ is at most $\frac{4}{5} \mathrm{Opt}(P)+1$. Since by Yao's principle, $C_{\mathrm{R}}^{\mathrm{PATH}}(2) \leq \mathbb{E}[\mathrm{D}(P)] / \mathrm{OPT}(P)$ (and $\mathbb{E}[\mathrm{D}(P)]$ can be arbitrarily large), this will complete the proof.

We first introduce some terminology to describe a coloring produced by $\mathrm{D}$. For any $i, 2 \leq i \leq M+1$, we say that $e_{i-1}$ is the previous isolated edge of $e_{i}$. The set of isolated edges is partitioned into the following four sets:

$E^{\mathrm{s}}$ : Isolated edges colored with the same color as the previous isolated edge.

$E^{\text {d: }}$ Isolated edges colored differently from the previous isolated edge.

$E^{\mathrm{r}}$ : Isolated edges that are rejected.

$E^{\mathrm{c}}$ : Isolated edges that are colored but whose previous isolated edge is rejected.

Clearly, $\left|E^{\mathrm{c}}\right| \leq\left|E^{\mathrm{r}}\right|$.

Let $X$ be a random variable denoting the total number of edges rejected by $\mathrm{D}$. We will give a lower bound on $\mathbb{E}[X]$. For each isolated edge $e_{i}$ with $2 \leq i \leq M+1$, consider the probability of at least one of $e_{i}$ and the edge(s) connecting $e_{i}$ to $e_{i-1}$ being rejected. For each edge in $E^{\mathrm{s}}$, the algorithm $\mathrm{D}$ makes a rejection with probability $\frac{1}{2}$, since it will be forced to do so if $i \in$ $\bar{S}$. Conversely, for each edge in $E^{\mathrm{d}}$, the algorithm D makes a rejection with probability $\frac{1}{2}$, since it is forced to do so if $i \in S$. Also, for each edge in $E^{\mathrm{r}}$, the algorithm D makes a rejection with probability 1 . Combining these observations with the linearity of expectation, we get that

$$
\begin{aligned}
\mathbb{E}[X] & \geq\left(\frac{1}{2}\left|E^{\mathrm{s}}\right|+\frac{1}{2}\left|E^{\mathrm{d}}\right|+\left|E^{\mathrm{r}}\right|\right) \\
& \geq \frac{1}{2}\left(\left|E^{\mathrm{s}}\right|+\left|E^{\mathrm{d}}\right|+\left|E^{\mathrm{r}}\right|+\left|E^{\mathrm{c}}\right|\right), \text { since }\left|E^{\mathrm{r}}\right| \geq\left|E^{\mathrm{c}}\right| \\
& \geq \frac{M}{2} .
\end{aligned}
$$

Finally, since Opт can color all $\frac{5}{2} M+1$ edges of the path, we get that

$$
\mathbb{E}[\mathrm{D}(P)] \leq 2 M+1<\frac{4}{5} \operatorname{OpT}(P)+1 .
$$

Since $M$ can be arbitrarily large, this proves the theorem. 
Theorems 1 and 2 together give the following corollary.

Corollary 1 For $p=\frac{\varphi}{\sqrt{5}}, \mathrm{RAND}_{p}$ is optimal for EDGe-2-ColoRIng(PATH) with

$$
C_{\mathrm{RAND}_{p}}^{\mathrm{PATH}}(2)=\frac{4}{5}
$$

\section{Coloring of Trees}

We will now consider the EDGE- $k$-COLORING problem when the input graph is a tree. Our main result is a proof that FIRST-FIT is optimal among deterministic as well as fair algorithms. We also show that even randomized algorithms that are not fair can only be slightly better that FirsT-FiT. Finally, we show that, for any fixed $k \geq 4$, FIRST-FIT has a better competitive ratio than Next-Fit.

First, we give a general upper bound for algorithms that are deterministic and/or fair.

Theorem 3 If $\mathrm{A}$ is a deterministic or fair algorithm and $k \geq 2$, then

$$
C_{\mathrm{A}}^{\mathrm{TREE}}(k) \leq \frac{k-1}{k} .
$$

Proof The adversary reveals the edges of a tree in $N$ steps, for some large $N \in \mathbb{N}$. The set of edges revealed in the $i$ th step constitute a star, $S_{i}$, with $k+1$ edges and center vertex $c_{i}$. If at least one edge in $S_{i-1}$ is colored, the adversary chooses $c_{i}=x$ for some colored edge $\left(c_{i-1}, x\right)$ in $S_{i-1}$. Otherwise, it chooses $c_{i}=x$ for an arbitrary edge $\left(c_{i-1}, x\right)$ in $S_{i-1}$. Note that the adversary is clearly able to identify a colored edge in $S_{i-1}$, if one exists: If A is deterministic, this is trivially true, and if A is fair, the first $k-1$ edges of $S_{i-1}$ will be colored.

The algorithm A may color $k$ edges of $S_{1}$. For all other values of $i$, there are two possibilities:

- If A colors even a single edge of $S_{i-1}$, then it can color at most $k-1$ edges of $S_{i}$.

- Even if A rejects all edges of $S_{i-1}$, then it can color at most $k$ edges of $S_{i}$.

Let $N_{0}$ denote the number of stars where A colors no edges. Then, A colors at $\operatorname{most}\left(N_{0}+1\right) k+\left(N-2 N_{0}-1\right)(k-1)=N(k-1)-(k-2) N_{0}+1 \leq N(k-1)+1$ edges. On the other hand, in each star, Opt colors the $k$ edges not incident to other stars, in total $N k$ edges. Since $N$ can be arbitrarily large, this shows that the competitive ratio of $\mathrm{A}$ is at most $\frac{k-1}{k}$.

Using the charging technique of Section 2, we will show that Theorem 3 is tight by proving a matching lower bound for FIRST-FIT. To this end, we introduce some terminology related to deterministic algorithms.

Let A be a deterministic algorithm for EdGE- $k$-Coloring, let $G=(V, E)$ be a graph, and suppose that A has been given the edges of $G$ in some order. Recall that, since $\mathrm{A}$ is deterministic, $E_{+}$denotes the set of edges colored by A, 
and $E_{-}$denotes the set of edges colored by OPT only. We partition $E_{+}$into the set, $E_{+}^{\mathrm{d}}$, of edges colored by both A and Opt (double colored edges) and the set, $E_{+}^{\mathrm{s}}$, of edges colored by A only (single colored edges). Thus, $E_{\text {Орт }}=E_{-} \cup E_{+}^{\mathrm{d}}$. For $x \in V$, let $E_{+}(x)$ be the edges in $E_{+}$incident to $x$ and let $d_{+}(x)=\left|E_{+}(x)\right|$. Define $E_{-}(x), E_{+}^{\mathrm{d}}(x), E_{+}^{\mathrm{s}}(x), d_{-}(x), d_{+}^{\mathrm{d}}(x)$ and $d_{+}^{\mathrm{s}}(x)$ similarly.

Theorem 4 For $k \geq 2$, FIRST-FIT is an optimal deterministic algorithm for EdGe- $k$-Coloring(Tree) with

$$
C_{\mathrm{FF}}^{\mathrm{TREE}}(k)=\frac{k-1}{k}
$$

Proof Fix a tree $T=(V, E)$ and assume that the edges of $E$ have been revealed to First-Fit in some order. For the analysis, we will view $T$ as a rooted tree by choosing an arbitrary vertex to be the root. When writing $e=(x, y) \in E$, we imply that $x$ is the parent vertex of $y$.

Following Section 2, we set $C=\frac{k-1}{k}$. An edge in $E_{+}^{\mathrm{d}}$ then has a surplus of $1-C=\frac{1}{k}$ and an edge in $E_{+}^{\mathrm{s}}$ has a surplus of 1 . On the other hand, an edge in $E_{-}$has an initial value of zero.

We will define a strategy to distribute the total positive surplus obtained by FIRST-Fit among the edges in $E_{-}$such that each edge gets a final value of at least $C$. For ease of presentation, the strategy will be described in a stepwise manner (see Fig. 1 for an illustration of how the strategy works):

Step 1: Consider in turn all edges $e=(v, u) \in E_{+}$. Let $c$ be the color assigned to $e$ by First-Fit and let $e^{\prime}=(w, v)$ be the parent edge of $e$ (if it exists).

(a) If $e^{\prime} \in E_{+}^{\mathrm{d}}$ and $e^{\prime}$ has been colored with a color $c^{\prime}>c$, then $e$ transfers a value of $\frac{1}{k}$ to $w$.

(b) Any surplus remaining at $e$ is transferred to $v$.

For each vertex $v$, let $m(v)$ denote the value transferred to $v$ in this step.

Step 2: Consider in turn all vertices $v \in V$.

(a) If the vertex $v$ has a parent edge $e^{\prime} \in E_{-}$, then $v$ transfers a value of $\min \left\{m(v), \frac{k-1}{k}\right\}$ to $e^{\prime}$.

(b) Any value remaining at $v$ is distributed equally among the child edges of $v$ belonging to $E_{-}$.

For each edge $e$, let $m_{v}(e)$ denote the value transferred from $v$ to $e$ in this step.

The following simple but useful properties of the strategy defined above will be used to prove the theorem. Each of the four facts gives a lower bound on the value transferred from an edge $e_{+}=(v, u) \in E_{+}$to its parent vertex, $v$. Let $c$ denote the color of $e_{+}$. We first state the four facts and then give short proofs.

Let $e^{\prime}=(w, v)$ be the parent edge of $e_{+}$(if it exists). If $e^{\prime} \in E_{+}$, let $c^{\prime}$ denote the color of $e^{\prime}$. 


$E_{+}^{s} \overline{E_{+}^{d}} \quad \overline{E_{-}^{-}}$
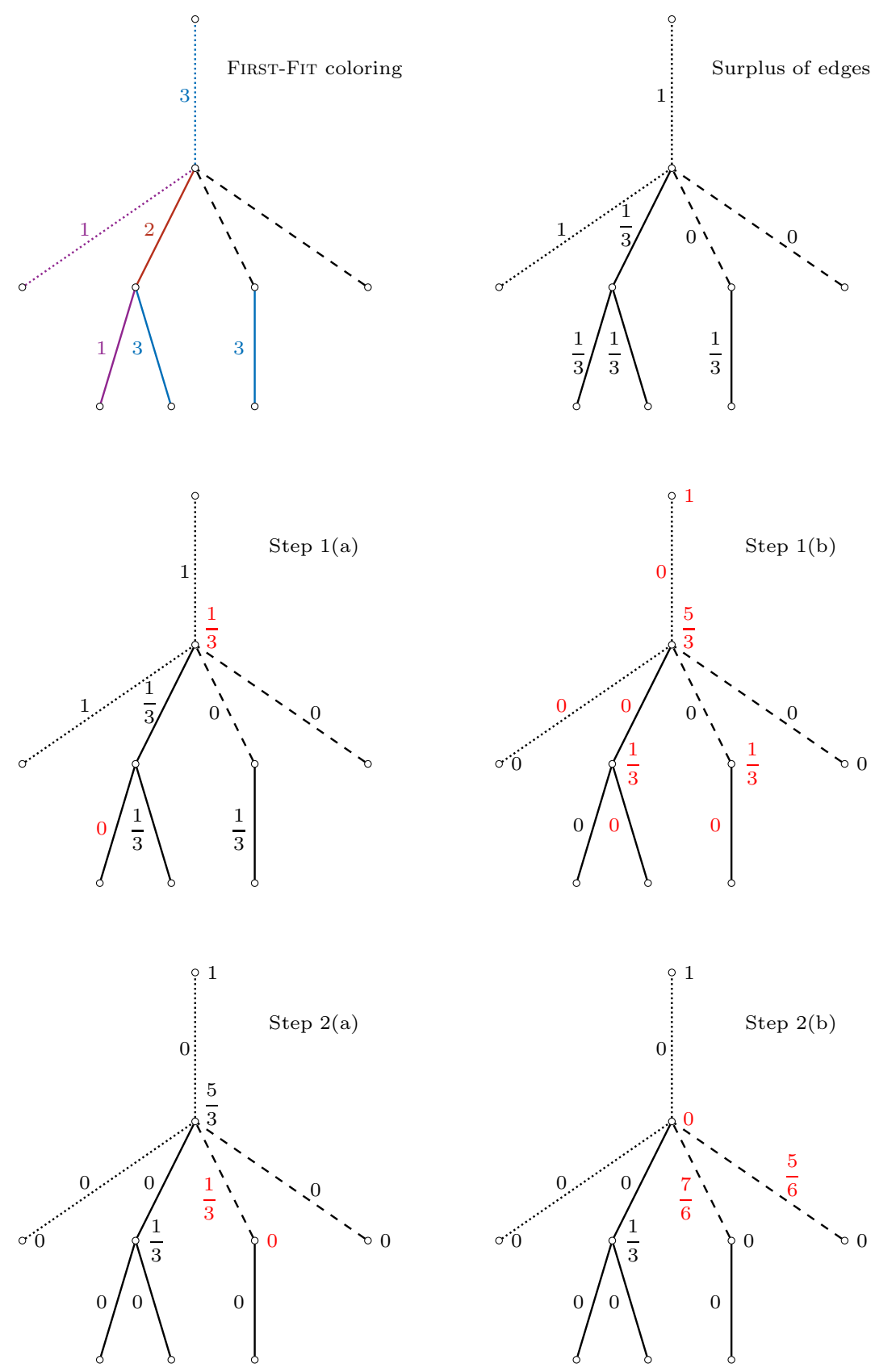

Fig. 1 Illustration of the steps of the strategy defined in the proof of Theorem 4 In this example, the number of colors is $k=3$. 
Fact 1: Assume that $e_{+} \in E_{+}^{\mathrm{s}}$.

If $e^{\prime} \notin E_{+}^{\mathrm{d}}, e^{\prime}$ does not exist, or $c \geq c^{\prime}$, then $e_{+}$contributes a value of 1 to $m(v)$.

If $e^{\prime} \in E_{+}^{\mathrm{d}}$, then $e_{+}$contributes a value of at least $\frac{k-1}{k}$ to $m(v)$.

Fact 2: If $e^{\prime} \notin E_{+}$or $e^{\prime}$ does not exist, then $m(v) \geq \frac{c}{k}$.

Fact 3: Assume that $e_{+} \in E_{+}^{\mathrm{d}}$.

If $e^{\prime} \notin E_{+}^{\mathrm{d}}$, then $e_{+}$contributes a value of $\frac{1}{k}$ to $m(v)$.

In order to state the next fact, we need to introduce some new terminology. For $v \in V$, let $\widehat{c}_{v}=\max \left(\overline{\mathcal{C}_{v}} \cup\{0\}\right)$. That is, $\widehat{c_{v}}$ is the largest color available at $v$ (and $\widehat{c}_{v}=0$ if no colors are available). If an edge incident to $v$ is colored with a color $c>\widehat{c}_{v}$, the edge is said to be a high-colored edge (with respect to $v$ ). There must be exactly $k-\widehat{c}_{v}$ high-colored edges incident to $v$.

Fact 4: Assume that $e_{+} \in E_{+}^{\mathrm{d}}$.

If $e_{+}$is high-colored with respect to $v$, then the colored child edges of $e_{+}$contribute a total value of at least $\frac{k-d_{+}(v)}{k}$ to $m(v)$.

Proof of Fact 1: If $e^{\prime} \notin E_{+}^{\mathrm{d}}, e^{\prime}$ does not exists, or $c \geq c^{\prime}$, then $e_{+}$transfers a value of 1 to $v$ in Step 1(b). If $e^{\prime} \in E_{+}^{\mathrm{d}}$, then $e_{+}$transfers a value of at most $\frac{1}{k}$ to $w$ in Step 1(a) and hence $e_{+}$transfers a value of at least $\frac{k-1}{k}$ to $v$ in Step $1(\mathrm{~b})$.

Proof of Fact 2: If $e_{+} \in E_{+}^{\mathrm{s}}$, this follows from Fact 1. Otherwise, note that by the definition of FIRST-FIT, it must hold that $\mathcal{C}_{1, c} \subseteq \mathcal{C}_{v} \cup \mathcal{C}_{u}$. In Step 1, the edges incident to $v$ and $u$ colored with a color in $\mathcal{C}_{1, c}$ each transfer a value of at least $\frac{1}{k}$ to $v$.

Proof of Fact 3: This follows, since $e_{+}$does not transfer any value to $w$ in Step 1(a).

Proof of Fact 4: Since $e_{+}$is high-colored, it follows from the definition of First-Fit that all colors in $\overline{\mathcal{C}_{v}}$ are represented at child edges of $u$. Thus, $e_{+}$ has at least $\left|\overline{\mathcal{C}_{v}}\right|=k-d_{+}(v)$ child edges with lower colors than the color of $e_{+}$. Since $e_{+} \in E_{+}^{\mathrm{d}}$, each of these child edges transfers a value of $\frac{1}{k}$ to $v$ in Step $1(\mathrm{a})$.

We will combine these facts to show that any edge $e=(x, y) \in E_{-}$gets a final value of at least $\frac{k-1}{k}$.

If $\mathcal{C}_{x}=\mathcal{C}_{1, k}$, then $\widehat{c}_{x}=0$. Otherwise, $\widehat{c}_{x} \in \mathcal{C}_{y}$, since FIRST-FIT is fair. Hence, Fact 2 implies that $m(y) \geq \frac{\widehat{c}_{x}}{k}$. Thus, $e$ receives a value of at least $\min \left\{\frac{k-1}{k}, \frac{\widehat{c}_{x}}{k}\right\}$ from $y$. In particular, we will assume that $\widehat{c}_{x}<k-1$, since otherwise we are done. Thus,

$$
m_{y}(e) \geq \frac{\widehat{c}_{x}}{k}
$$

We will now turn to proving that $m_{x}(e) \geq \frac{k-\widehat{c}_{x}-1}{k}$. This will finish the proof, since it means that $e$ gets a final value of $m_{x}(e)+m_{y}(e) \geq \frac{k-\widehat{c}_{x}-1}{k}+\frac{\widehat{c}_{x}}{k}=\frac{k-1}{k}$.

Let $e^{\prime}=(z, x)$ be the parent edge of $x$ (if it exists). The rest of the proof is split into three cases depending on which of the sets $E_{+}^{\mathrm{d}}, E_{+}^{\mathrm{s}}$, and $E_{-}$(if any) that contains $e^{\prime}$. 
Case 1: $e^{\prime} \in E_{+}^{d}$. Recall that there are $k-\widehat{c}_{x}$ high-colored edges incident to $x$. Thus, $x$ has at least $k-\widehat{c}_{x}-1$ high-colored child edges, and at least $k-\widehat{c}_{x}-1-d_{+}^{\mathrm{s}}(x)$ of them belong to $E_{+}^{\mathrm{d}}$. By Fact $4, x$ receives a value of at least $\frac{k-d_{+}(x)}{k}$ from the child edges of each of these at least $k-\widehat{c}_{x}-1-d_{+}^{\mathrm{s}}(x)$ edges. Moreover, by Fact 1 , each of the $d_{+}^{\mathrm{s}}(x)$ child edges of $e^{\prime}$ belonging to $E_{+}^{\mathrm{s}}$ contributes a value of $\frac{k-1}{k}$ to $m(x)$. Thus,

$$
\begin{aligned}
m(x) & \geq\left(k-\widehat{c}_{x}-1-d_{+}^{\mathrm{s}}(x)\right) \frac{k-d_{+}(x)}{k}+d_{+}^{\mathrm{s}}(x) \frac{k-1}{k} \\
& =\left(k-\widehat{c}_{x}-1-d_{+}^{\mathrm{s}}(x)\right) \frac{k-d_{+}(x)}{k}+d_{+}^{\mathrm{s}}(x)\left(\frac{k-d_{+}(x)}{k}+\frac{d_{+}(x)-1}{k}\right) \\
& =\left(k-\widehat{c}_{x}-1\right) \frac{k-d_{+}(x)}{k}+d_{+}^{\mathrm{s}}(x) \frac{d_{+}(x)-1}{k} \\
& \geq\left(k-\widehat{c}_{x}-1\right) \frac{k-d_{+}(x)}{k}+d_{+}^{\mathrm{s}}(x) \frac{k-\widehat{c}_{x}-1}{k} \\
& =\left(k-d_{+}(x)+d_{+}^{\mathrm{s}}(x)\right) \frac{k-\widehat{c}_{x}-1}{k} \\
& =\left(k-d_{+}^{\mathrm{d}}(x)\right) \frac{k-\widehat{c}_{x}-1}{k} \\
& \geq d_{-}(x) \frac{k-\widehat{c}_{x}-1}{k}
\end{aligned}
$$

Hence, since no value is transferred from $x$ to $e^{\prime}$ in Step 2(a), each child edge of $x$ belonging to $E_{-}$receives a value of at least $\frac{k-\widehat{c}_{x}-1}{k}$ from $x$ in Step 2(b). In particular,

$$
m_{x}(e) \geq \frac{k-\widehat{c}_{x}-1}{k}
$$

Case 2: $e^{\prime} \in E_{+}^{s}$ or $e^{\prime}$ does not exist. In this case, since $e^{\prime} \notin E_{+}^{\mathrm{d}}, x$ has at least $k-\widehat{c}_{x}-d_{+}^{\mathrm{s}}(x)$ high-colored child edges belonging to $E_{+}^{\mathrm{d}}$. By Fact $4, x$ receives a value of at least $\frac{k-d_{+}(x)}{k}$ from the child edges of each of these edges. Note that this value comes solely from child edges of $x$ 's high-colored child edges, not from the high-colored edges themselves. Therefore, by Fact 3, there is also a contribution of $\frac{1}{k}$ from each of $x$ 's child edges belonging to $E_{+}^{\mathrm{d}}$. Finally, there are at least $d_{+}^{\mathrm{s}}(x)-1$ child edges of $x$ belonging to $E_{+}^{\mathrm{s}}$ (if $e^{\prime}$ exists, there are $d_{+}^{\mathrm{s}}(x)-1$ such edges, and otherwise there are $d_{+}^{\mathrm{s}}(x)$ such edges). By Fact 1 , 
each of these edges transfers a value of 1 to $x$. Thus,

$$
\begin{aligned}
m(x) \geq & \left(k-\widehat{c}_{x}-d_{+}^{\mathrm{s}}(x)\right) \frac{k-d_{+}(x)}{k}+\left(d_{+}^{\mathrm{s}}(x)-1\right)+\frac{d_{+}^{\mathrm{d}}(x)}{k} \\
= & \left(k-\widehat{c}_{x}-d_{+}^{\mathrm{s}}(x)\right) \frac{k-d_{+}(x)}{k}+\left(d_{+}^{\mathrm{s}}(x)-1\right)\left(\frac{k-d_{+}(x)}{k}+\frac{d_{+}(x)}{k}\right)+\frac{d_{+}^{\mathrm{d}}(x)}{k} \\
= & \left(k-\widehat{c}_{x}\right) \frac{k-d_{+}(x)}{k}-d_{+}^{\mathrm{s}}(x) \frac{k-d_{+}(x)}{k}+d_{+}^{\mathrm{s}}(x) \frac{k-d_{+}(x)}{k}-\frac{k-d_{+}(x)}{k} \\
& +\left(d_{+}^{\mathrm{s}}(x)-1\right) \frac{d_{+}(x)}{k}+\frac{d_{+}^{\mathrm{d}}(x)}{k} \\
= & \left(k-\widehat{c}_{x}-1\right) \frac{k-d_{+}(x)}{k}+\left(d_{+}^{\mathrm{s}}(x)-1\right) \frac{d_{+}(x)}{k}+\frac{d_{+}^{\mathrm{d}}(x)}{k} \\
= & \left(k-\widehat{c}_{x}-1\right) \frac{k-d_{+}(x)}{k}+\left(d_{+}^{\mathrm{s}}(x)-1\right) \frac{d_{+}(x)-1}{k}+\frac{d_{+}^{\mathrm{s}}(x)-1+d_{+}^{\mathrm{d}}(x)}{k} \\
= & \left(k-\widehat{c}_{x}-1\right) \frac{k-d_{+}(x)}{k}+\left(d_{+}^{\mathrm{s}}(x)-1\right) \frac{d_{+}(x)-1}{k}+\frac{d_{+}(x)-1}{k} \\
= & \left(k-\widehat{c}_{x}-1\right) \frac{k-d_{+}(x)}{k}+d_{+}^{\mathrm{s}}(x) \frac{d_{+}(x)-1}{k} \\
\geq & d_{-}(x) \frac{k-\widehat{c}_{x}-1}{k}, \text { as in Case } 1
\end{aligned}
$$

Hence, since no value is transferred from $x$ to $e^{\prime}$ in Step 2(a), each child edge of $x$ belonging to $E_{-}$receives a value of at least $\frac{k-\widehat{c}_{x}-1}{k}$ from $x$ in Step 2(b). Thus,

$$
m_{x}(e) \geq \frac{k-\widehat{c}_{x}-1}{k}
$$

Case 3: $e^{\prime} \in E_{-}$. The only difference to Case 2 is that $x$ has exactly $d_{+}^{\mathrm{s}}(x)$ child edges belonging to $E_{+}^{\mathrm{s}}$. Thus,

$$
\begin{aligned}
m(x) & \geq\left(k-\widehat{c}_{x}-d_{+}^{\mathrm{s}}(x)\right) \frac{k-d_{+}(x)}{k}+d_{+}^{\mathrm{s}}(x)+\frac{d_{+}^{\mathrm{d}}(x)}{k} \\
& \geq d_{-}(x) \frac{k-\widehat{c}_{x}-1}{k}+1, \text { using the same calculations as in Case } 2
\end{aligned}
$$

Hence, since the value transferred from $x$ to $e^{\prime}$ is smaller than 1, each child edge of $x$ belonging to $E_{-}$receives a value larger than $\frac{k-\widehat{c}_{x}-1}{k}$ from $x$. Thus, again,

$$
m_{x}(e) \geq \frac{k-\widehat{c}_{x}-1}{k}
$$

By Theorems 3 and 4, an algorithm for Edge- $k$-Coloring(Tree) can only be better than FIRST-FiT, if it is both randomized and unfair. However, the next result shows that even such algorithms cannot do much better than FIRST-FIT. 
Theorem 5 If $\mathrm{R}$ is a (possibly randomized) algorithm for EDGE- $k$-COLORING and $k \geq 2$, then

$$
C_{\mathrm{R}}^{\mathrm{TREE}}(k) \leq \frac{k}{k+1}
$$

Proof The adversary first reveals the edges of a path $P=\left\langle e_{1}, \ldots, e_{m}\right\rangle$, for some large $m \in \mathbb{N}$. Let $v_{1}, \ldots, v_{m+1}$ be the vertices in the path such that $e_{i}=\left(v_{i}, v_{i+1}\right)$, for $1 \leq i \leq m$. If $\mathbb{E}[\mathrm{R}(P)] \leq \frac{k}{k+1} m$, the adversary reveals no more edges. If $\mathbb{E}[\mathrm{R}(P)]>\frac{k}{k+1} m$, then for each $i, 1 \leq i \leq m+1$, the adversary reveals $k$ edges constituting a star, $S_{i}$, with center vertex $v_{i}$. Let $S$ be the set consisting of the edges of every star $S_{i}$ for $1 \leq i \leq m+1$.

If the adversary only reveals the edges of the path $P$, then $\mathbb{E}[\mathrm{R}(P)] \leq \frac{k}{k+1} m$ and so $\mathbb{E}[\mathrm{R}(P)] \leq \frac{k}{k+1} \mathrm{OPT}(P)$. Indeed, Opt can color all $m$ edges in $P$, since $k \geq 2$ and so $\operatorname{OPT}(P)=m$. Assume now that the adversary also reveals the stars. In this case, OPT rejects all edges of the path and instead colors the $k$ edges of each star. Thus, $\operatorname{Opt}(P \cup S)=k(m+1)$. Note that each of the edges $e_{i}=\left(v_{i}, v_{i+1}\right)$ is incident to the center vertices of both $S_{i}$ and $S_{i+1}$. This implies that $\mathbb{E}[\mathrm{R}(S)] \leq k(m+1)-2 \mathbb{E}[\mathrm{R}(P)]$. Using the assumption $\mathbb{E}[\mathrm{R}(P)]>\frac{k}{k+1} m$, we get that

$$
\begin{aligned}
\mathbb{E}[\mathrm{R}(P \cup S)] & =\mathbb{E}[\mathrm{R}(P)]+\mathbb{E}[\mathrm{R}(S)] \\
& \leq \mathbb{E}[\mathrm{R}(P)]+k(m+1)-2 \mathbb{E}[\mathrm{R}(P)] \\
& \leq k(m+1)-\frac{k}{k+1} m \\
& =\frac{k(k m+k+1)}{k+1} \\
& =\frac{k}{k+1} k(m+1)+\frac{k}{k+1} \\
& =\frac{k}{k+1} \operatorname{OpT}(P \cup S)+\frac{k}{k+1}
\end{aligned}
$$

Since $m$ can be arbitrarily large, this shows that $\mathrm{R}$ cannot be better than $\frac{k}{k+1}$-competitive.

We now show that the competitive ratio of any fair algorithm tends to 1 as $k$ tends to infinity.

Theorem 6 If $\mathrm{F}$ is a fair algorithm, then for any $k \geq 2$,

$$
C_{\mathrm{F}}^{\mathrm{TREE}}(k) \geq \frac{2 \sqrt{k}-2}{2 \sqrt{k}-1} .
$$

Proof Assume first that $\mathrm{F}$ is a deterministic algorithm. Let $T=(V, E)$ be a tree and assume that the edges of $T$ have been revealed to $F$ in some order. For the analysis, we will view $T$ as a rooted tree by choosing an arbitrary vertex to be the root. As in the proof of Theorem 4, we let $e=(x, y)$ imply that $x$ is the parent of $y$. 
We will apply the charging technique from Section 2 to show that $\mathrm{F}$ is $C$-competitive, where $C=\frac{2 \sqrt{k}-2}{2 \sqrt{k}-1}$. We will use the notation introduced just before Theorem 4, Recall that all edges in $E_{+}$have an initial value of 1 . Edges in $E_{+}^{\mathrm{d}}$ have a surplus of $1-C$ and edges in $E_{+}^{\mathrm{s}}$ have a surplus of 1 . Edges in $E_{-}$have an initial value of 0 . The goal is to distribute the surplus from $E_{+}$ among the edges in $E_{-}$so that all of them get a final value of at least $C$. To this end, we use the following strategy:

Step 1: Each edge $(v, u) \in E_{+}$transfers its surplus to its parent vertex, $v$. For each vertex $v$, let $m(v)$ denote the value transferred to $v$ in this step.

Step 2: Consider in turn all vertices $v \in V$.

(a) If the vertex $v$ has a parent edge $e^{\prime} \in E_{-}$, then $v$ transfers a value of $\min \{m(v), C\}$ to $e^{\prime}$.

(b) Any value remaining at $v$ is distributed equally among the child edges of $v$ belonging to $E_{-}$.

For each edge $e$, let $m_{v}(e)$ denote the value transferred from $v$ to $e$ in this step.

This finishes the description of the strategy.

Fix an edge $e=(x, y) \in E_{-}$. In Step 1, $y$ receives $m(y)=d_{+}(y)-C d_{+}^{\mathrm{d}}(y)$. Thus, in Step 2(a), $e$ receives

$$
m_{y}(e)=\min \left\{C, d_{+}(y)-C d_{+}^{\mathrm{d}}(y)\right\}
$$

from $y$. We will show that $m_{x}(e)+m_{y}(e) \geq C$. If $m_{y}(e) \geq C$, this is clearly true. Thus, we may assume that $d_{+}(y)-C d_{+}^{\mathrm{d}}(y)<C$. Note that

$$
\begin{aligned}
d_{+}(y)-C d_{+}^{\mathrm{d}}(y)<C & \Rightarrow d_{+}(y)<C\left(d_{+}^{\mathrm{d}}(y)+1\right)<d_{+}^{\mathrm{d}}(y)+1 \\
& \Rightarrow d_{+}(y)-d_{+}^{\mathrm{d}}(y)<1 \\
& \Rightarrow d_{+}(y)=d_{+}^{\mathrm{d}}(y) .
\end{aligned}
$$

It follows that we only need to consider the case where $d_{+}(y)=d_{+}^{\mathrm{d}}(y)$, meaning that all of the edges incident to $y$ which have been colored by $\mathrm{F}$ have also been colored by OPT. This implies that the value transferred to $e$ from its colored child edges is

$$
m_{y}(e)=(1-C) d_{+}(y) .
$$

When calculating a lower bound on $m_{x}(e)$, we consider four cases. In each case, we use the following two simple facts.

Fact 1: $d_{+}^{\mathrm{d}}(x)+d_{-}(x) \leq k$.

Fact 2: $d_{+}(x)+d_{+}(y) \geq k$.

Proof of Fact 1: Note that $d_{+}^{\mathrm{d}}(x)+d_{-}(x)$ is exactly the number of edges incident to $x$ that are colored by OpT. Thus, Fact 1 follows trivially, since no algorithm can color more than $k$ edges incident to $x$. 
Proof of Fact 2: This follows from the fact that the edge $(x, y)$ is rejected by the fair algorithm $\mathrm{F}$.

In what follows, we will rely on the following elementary fact: Consider a quadratic polynomial $a x^{2}+b x+c$ with $a, b, c \in \mathbb{R}$ and $a>0$. If the discriminant $D=b^{2}-4 a c=0$, then the polynomial is non-negative.

Case 1: The parent edge of $x$ belongs to $E_{-}$. In this case,

$$
\begin{aligned}
m_{x}(e) & \geq \frac{m(x)-C}{d_{-}(x)-1}=\frac{d_{+}(x)-C d_{+}^{\mathrm{d}}(x)-C}{d_{-}(x)-1} \\
& \geq \frac{d_{+}(x)-C d_{+}^{\mathrm{d}}(x)-C}{k-d_{+}^{\mathrm{d}}(x)-1}, \text { by Fact } 1 .
\end{aligned}
$$

Thus, we obtain the following, where the second inequality follows from Fact 2 , and the third inequality comes from $d_{+}^{\mathrm{d}}(x) \leq d_{+}(x)$ :

$$
\begin{aligned}
m_{x}(e) & +m_{y}(e) \geq \frac{d_{+}(x)-C d_{+}^{\mathrm{d}}(x)-C}{k-d_{+}^{\mathrm{d}}(x)-1}+(1-C) d_{+}(y) \\
& \geq \frac{d_{+}(x)-C d_{+}^{\mathrm{d}}(x)-C}{k-d_{+}^{\mathrm{d}}(x)-1}+(1-C)\left(k-d_{+}(x)\right) \\
& =\frac{d_{+}(x)-C d_{+}^{\mathrm{d}}(x)-C+\left(k-d_{+}^{\mathrm{d}}(x)-1\right)(1-C)\left(k-d_{+}(x)\right)}{k-d_{+}^{\mathrm{d}}(x)-1} \\
& \geq \frac{d_{+}(x)-C d_{+}^{\mathrm{d}}(x)-C+\left(k-d_{+}(x)-1\right)(1-C)\left(k-d_{+}(x)\right)}{k-d_{+}^{\mathrm{d}}(x)-1} \\
& =\frac{d_{+}(x)-C d_{+}^{\mathrm{d}}(x)-C+(1-C)\left(k-d_{+}(x)\right)^{2}+(C-1)\left(k-d_{+}(x)\right)}{k-d_{+}^{\mathrm{d}}(x)-1} \\
& =\frac{(1-C)\left(k-d_{+}(x)\right)^{2}+(C-2)\left(k-d_{+}(x)\right)+(1-C) k}{k-d_{+}^{\mathrm{d}}(x)-1}+C \\
& \geq C .
\end{aligned}
$$

Here, the final inequality (2) holds since the numerator of the fraction is a quadratic polynomial in $\left(k-d_{+}(x)\right)$ whose discriminant is zero:

$$
(C-2)^{2}-4 \cdot(1-C) \cdot(1-C) k=\left(\frac{-2 \sqrt{k}}{2 \sqrt{k}-1}\right)^{2}-\frac{4 k}{(2 \sqrt{k}-1)^{2}}=0 .
$$

Case 2: The parent edge of $x$ belongs to $E_{+}^{s}$. In this case,

$$
\begin{aligned}
m_{x}(e) & =\frac{m(x)}{d_{-}(x)}=\frac{\left(d_{+}(x)-1\right)-C d_{+}^{\mathrm{d}}(x)}{d_{-}(x)} \\
& \geq \frac{d_{+}(x)-C d_{+}^{\mathrm{d}}(x)-1}{k-d_{+}^{\mathrm{d}}(x)}, \text { by Fact } 1 .
\end{aligned}
$$


Thus, we obtain the following, where the second inequality follows from Fact 2 and the third inequality comes from $d_{+}^{\mathrm{d}}(x)=d_{+}(x)-d_{+}^{\mathrm{s}}(x) \leq d_{+}(x)-1$ :

$$
\begin{aligned}
m_{x}(e)+m_{y}(e) & \geq \frac{d_{+}(x)-C d_{+}^{\mathrm{d}}(x)-1}{k-d_{+}^{\mathrm{d}}(x)}+(1-C) d_{+}(y) \\
& \geq \frac{d_{+}(x)-C d_{+}^{\mathrm{d}}(x)-1}{k-d_{+}^{\mathrm{d}}(x)}+(1-C)\left(k-d_{+}(x)\right) \\
& =\frac{d_{+}(x)-C d_{+}^{\mathrm{d}}(x)-1+(1-C)\left(k-d_{+}(x)\right)\left(k-d_{+}^{\mathrm{d}}(x)\right)}{k-d_{+}^{\mathrm{d}}(x)} \\
& \geq \frac{d_{+}(x)-C d_{+}^{\mathrm{d}}(x)-1+(1-C)\left(k-d_{+}(x)\right)\left(k-d_{+}(x)+1\right)}{k-d_{+}^{\mathrm{d}}(x)} \\
& =\frac{(1-C)\left(k-d_{+}(x)\right)^{2}-C\left(k-d_{+}(x)\right)+k-1-C d_{+}^{\mathrm{d}}(x)}{k-d_{+}^{\mathrm{d}}(x)} \\
& =\frac{(1-C)\left(k-d_{+}(x)\right)^{2}-C\left(k-d_{+}(x)\right)+(1-C) k-1}{k-d_{+}^{\mathrm{d}}(x)}+C \\
& \geq C .
\end{aligned}
$$

Here, the final inequality (3) holds since the numerator of the fraction is a quadratic polynomial in $\left(k-d_{+}(x)\right)$ whose discriminant is zero:

$$
\begin{aligned}
C^{2}-4(1-C)((1-C) k-1) & =C^{2}-4 \frac{1}{2 \sqrt{k}-1}\left(\frac{(\sqrt{k}-1)^{2}}{2 \sqrt{k}-1}\right) \\
& =C^{2}-4 \frac{(\sqrt{k}-1)^{2}}{(2 \sqrt{k}-1)^{2}}=0 .
\end{aligned}
$$

Case 3: The parent edge of $x$ belongs to $E_{+}^{d}$. In this case, we have

$$
\begin{aligned}
m_{x}(e) & =\frac{m(x)}{d_{-}(x)}=\frac{\left(d_{+}(x)-1\right)-C\left(d_{+}^{\mathrm{d}}(x)-1\right)}{d_{-}(x)} \\
& \geq \frac{d_{+}(x)-C d_{+}^{\mathrm{d}}(x)+C-1}{k-d_{+}^{\mathrm{d}}(x)}, \text { by Fact } 1 .
\end{aligned}
$$

Recall that $m_{y}(e)=d_{+}(y)(1-C)$. Thus, if $d_{+}(y)(1-C) \geq C$, we are done. Hence, we assume from now on that $d_{+}(y)<\frac{C}{1-C}=2 \sqrt{k}-2$. By Fact 2, this implies that $d_{+}(x)>k-(2 \sqrt{k}-2)$. Therefore, $(1-C)\left(k-d_{+}(x)\right)<$ $(1-C)(2 \sqrt{k}-2)=C$ which implies the second to last inequality below. The second inequality below follows from Fact 2 and the third inequality comes 


$$
\begin{aligned}
& \text { from } d_{+}^{\mathrm{d}}(x) \leq d_{+}(x) . \\
& \qquad \begin{aligned}
m_{x}(e)+m_{y}(e) & \geq \frac{d_{+}(x)-C d_{+}^{\mathrm{d}}(x)+C-1}{k-d_{+}^{\mathrm{d}}(x)}+(1-C) d_{+}(y) \\
& \geq \frac{d_{+}(x)-C d_{+}^{\mathrm{d}}(x)+C-1}{k-d_{+}^{\mathrm{d}}(x)}+(1-C)\left(k-d_{+}(x)\right), \text { by Fact } 2 \\
& =\frac{d_{+}(x)-C d_{+}^{\mathrm{d}}(x)+C-1+(1-C)\left(k-d_{+}(s)\right)\left(k-d_{+}^{\mathrm{d}}(x)\right)}{k-d_{+}^{\mathrm{d}}(x)} \\
& \geq \frac{d_{+}(x)-C d_{+}^{\mathrm{d}}(x)+C-1+(1-C)\left(k-d_{+}(x)\right)\left(k-d_{+}(x)\right)}{k-d_{+}^{\mathrm{d}}(x)} \\
& >\frac{d_{+}(x)-C d_{+}^{\mathrm{d}}(x)-1+(1-C)\left(k-d_{+}(x)\right)\left(k-d_{+}(x)+1\right)}{k-d_{+}^{\mathrm{d}}(x)} \\
& \geq C .
\end{aligned}
\end{aligned}
$$

Here, the final inequality (4) follows exactly as in Case 2.

Case 4: The parent edge of $x$ does not exist. In this case,

$$
m_{x}(e)=\frac{m(x)}{d_{-}(x)}=\frac{d_{+}(x)-C d_{+}^{\mathrm{d}}(x)}{d_{-}(x)}>\frac{d_{+}(x)-C d_{+}^{\mathrm{d}}(x)+C-1}{k-d_{+}^{\mathrm{d}}(x)} .
$$

Thus, $m_{x}(e)+m_{y}(e) \geq C$ follows as in Case 3 .

Randomized algorithms. Assume now that $\mathrm{F}$ is a randomized algorithm. The above analysis holds for any coloring that F may produce. Hence, for any coloring produced by $\mathrm{F}$, the number of colored edges is at least $(2 \sqrt{k}-2) /(2 \sqrt{k}-1)$ times the number of edges colored by OPT. Clearly, this means that the expected number of edges colored by $\mathrm{F}$ is at least $(2 \sqrt{k}-2) /(2 \sqrt{k}-1)$ times the number of edges colored by OpT.

We will show that the lower bound of Theorem [6] is essentially tight by providing a matching upper bound on the competitive ratio of NEXT-FIT when $k$ is a square number. To this end, we will use the following result from [9].

Lemma 3 (Favrholdt and Nielsen [9]) If the edges of a graph are colored in such a way that each color is used exactly $n$ or $n+1$ times for some $n \in \mathbb{N}$, then there exists an ordering of the edges such that NEXT-FIT produces an equivalent coloring.

The following corollary follows easily from Lemma 3

Corollary 2 Consider a graph, $G=(V, E)$, and a coloring, $\mathscr{C}$, of all edges of $G$ using at most $k$ colors. Let $H$ be a graph consisting of $k$ disjoint copies of $G$. There exists an ordering of the edges of $H$ such that, for each of the $k$ copies of $G$ in $H$, the coloring produced by NEXT-FIT is equivalent to $\mathscr{C}$. 
Proof Let $G_{1}, G_{2}, \ldots, G_{k}$ denote the $k$ copies of $G$. Furthermore, let $\mathscr{C}_{1}, \mathscr{C}_{2}$, $\ldots, \mathscr{C}_{k}$ be the $k$ colorings that can be obtained from $\mathscr{C}$ by cyclic permutations of the colors $1,2, \ldots, k$. If, for $1 \leq i \leq k, G_{i}$ is assigned the coloring $\mathscr{C}_{i}$, we obtain a coloring of $H$ where all colors are used the same number of times. The result now follows from Lemma 3 .

Note that Corollary 2 implies that if $\mathcal{G}$ is some family of graphs and $\mathcal{G}$ is closed under disjoint union, then NEXT-FIT has the worst possible competitive ratio among fair algorithms for $\operatorname{EDGE}-k$-Coloring $(\mathcal{G})$. This can be seen in the following way: For any graph, $G$, and any coloring, $\mathscr{C}$, of $G$ produced by a fair algorithm, the adversary can do the following:

- Make $k$ copies of $G$, resulting in a graph $H$.

- Give the edges of $H$ corresponding to the colored edges of $\mathscr{C}$. According to Corollary 2, these edges can be given in order, such that the edges of each copy of $G$ receives a coloring equivalent to $\mathscr{C}$.

- Give the edges of $H$ corresponding to edges that were not colored by $\mathscr{C}$. Since $\mathscr{C}$ was produced by a fair algorithm, NEXT-FIT will not be able to color any of these edges.

Hence, for any sequence, $E_{G}$, of edges and any fair algorithm $\mathrm{F}$, there is a sequence, $E_{H}$, of edges, such that NEXT-Fit uses just as many colors on $E_{H}$ as $\mathrm{F}$ does on $E_{G}$, and the optimal number of colors is the same for both sequences.

Even though TREE is not closed under disjoint union, a forest consisting of $k$ trees may be made into a single tree by revealing $k-1$ edges connecting the $k$ trees. Since this will add at most $k-1$ to the number of edges colored by NeXT-Fit, we may still apply Corollary 2 for the class TREE.

Theorem 7 For $k \geq 4$,

$$
C_{\mathrm{NF}}^{\mathrm{TREE}}(k) \leq \frac{\frac{k}{\lceil\sqrt{k}\rceil}+\lceil\sqrt{k}\rceil-2}{\frac{k}{\lceil\sqrt{k}\rceil}+\lceil\sqrt{k}\rceil-1} .
$$

In particular, if $k=n^{2}$ for some integer $n \geq 2$, then NEXT-FIT is a worst possible fair algorithm with

$$
C_{\mathrm{NF}}^{\mathrm{TREE}}(k)=\frac{2 \sqrt{k}-2}{2 \sqrt{k}-1} .
$$

Proof The lower bound for the case where $k$ is a square number follows from Theorem 6. For the upper bound, we define a tree $T=(V, E)$ and a subset $E^{\prime} \subset E$. We specify a coloring, $\mathscr{C}$, of $E^{\prime}$ with the property that each edge in $E \backslash E^{\prime}$ is adjacent to edges of all $k$ colors.

We first describe $E^{\prime}$ and $\mathscr{C}$. The tree $T$ contains $N$ bunches of stars, for some large $N$. Each bunch consists of a set of stars:

- One large star with $k-\lceil\sqrt{k}\rceil$ edges colored with $\mathcal{C}_{1, k-\lceil\sqrt{k}\rceil}$.

The center vertex of the large star in bunch $i, 1 \leq i \leq N$, is called $v_{i}$. 
- $\lceil\sqrt{k}\rceil-1$ small stars, each with $\lceil\sqrt{k}\rceil$ edges colored with $\mathcal{C}_{k-\lceil\sqrt{k}\rceil+1, k}$.

We now describe $E \backslash E^{\prime}$. For each $i, 1 \leq i \leq N, E \backslash E^{\prime}$ contains an edge between $v_{i}$ and the center vertex of each of the small stars in bunch $i$. For $1 \leq i<N$, there is an edge from $v_{i+1}$ to the center vertex of one of the small stars in the $i$ th bunch. Note that, after assigning the coloring $\mathscr{C}$ to $E^{\prime}$, none of the edges in $E \backslash E^{\prime}$ can be colored.

The adversary will use $k$ disjoint copies, $T_{1}=\left(V_{1}, E_{1}\right), \ldots, T_{k}=\left(V_{k}, E_{k}\right)$, of $T$. For each $T_{i}$, let $E_{i}^{\prime}$ denote the set of edges corresponding to $E^{\prime}$ and let $T_{i}^{\prime}=\left(V_{i}, E_{i}^{\prime}\right)$. If the edges of $E_{i}^{\prime} \cup E_{2}^{\prime} \cup \ldots \cup E_{k}^{\prime}$ are given first, it follows from Corollary 2 that they can be given an order such that the coloring produced by NeXT-Fit on each $T_{i}^{\prime}$ is equivalent to $\mathscr{C}$. Afterwards, no other edges can be colored.

Finally, the $k$ disjoint trees are connected, using $k-1$ edges between vertices that have degree one in the trees. The resulting tree is called $\mathcal{T}$.

Since $k \geq 4$, we must have $\lceil\sqrt{k}\rceil+2 \leq k$ and so the maximum degree of the graph is $k$. Thus, since the graph has no cycles, Opt colors all edges of the graph.

NEXT-Fit colors

$$
\begin{aligned}
\mathrm{NF}(\mathcal{T}) & =k N(k-\lceil\sqrt{k}\rceil+(\lceil\sqrt{k}\rceil-1)\lceil\sqrt{k}\rceil)+k-1 \\
& =k N\left(k+\lceil\sqrt{k}\rceil^{2}-2\lceil\sqrt{k}\rceil\right)+k-1
\end{aligned}
$$

edges and rejects $k(N(\lceil\sqrt{k}\rceil-1)+N-1)=k N\lceil\sqrt{k}\rceil-k$ edges. Since OPT colors all edges in the graph,

$$
\operatorname{Opt}(\mathcal{T})=k N\left(k+\lceil\sqrt{k}\rceil^{2}-\lceil\sqrt{k}\rceil\right)-1 .
$$

Thus,

$$
\begin{aligned}
\mathrm{NF}(\mathcal{T}) & \leq \frac{k+\lceil\sqrt{k}\rceil^{2}-2\lceil\sqrt{k}\rceil}{k+\lceil\sqrt{k}\rceil^{2}-\lceil\sqrt{k}\rceil} \operatorname{Opt}(\mathcal{T})+k \\
& =\frac{\frac{k}{\lceil\sqrt{k}\rceil}+\lceil\sqrt{k}\rceil-2}{\frac{k}{\lceil\sqrt{k}\rceil}+\lceil\sqrt{k}\rceil-1} \operatorname{OpT}(\mathcal{T})+k
\end{aligned}
$$

Since $N$ can be arbitrarily large, the result follows.

Theorem 7 shows that the bound of Theorem 6 is tight whenever $k$ is a square number. We will briefly consider the case where $k$ is not a square number. Any fair algorithm for EDGE-1-COLORING(TREE) is just the greedy matching algorithm. It is observed in several papers that this algorithm is $\frac{1}{2}$-competitive (for all input graphs) and that no deterministic algorithm can do better, even when the input graph is a tree. If $k \geq 2$, but not a square number, then the lower bound from Theorem 6 can be slightly improved by using the fact that $d_{+}(x)$ must be an integer. In particular, for $k=2$, it follows from Theorem 3.1 in [9] that any fair algorithm is $\frac{1}{2}$-competitive on any class 
of graphs. Combining this result with Theorem 3 shows that on trees, the competitive ratio of any fair algorithm is exactly $\frac{1}{2}$. We show in Theorems 8 and 9 that any fair algorithm for EDGE-3-ColorING(TREE) is $\frac{5}{8}$-competitive, and the competitive ratio of NEXT-FIT is exactly $\frac{5}{8}$. Thus, for $k \leq 4$, we have completely tight bounds. For $k \geq 5$, the difference between our upper and lower bounds is less than 0.0153 and tends to 0 as $k$ tends to infinity. Also, we get that FIRST-FIT has a strictly better competitive ratio than NEXT-FIT on trees whenever $k \geq 3$.

Theorem 8 If $\mathrm{F}$ is a fair algorithm, then $C_{\mathrm{F}}^{\mathrm{TREE}}(3) \geq \frac{5}{8}$.

Proof In order to prove that all fair algorithms are $\frac{5}{8}$-competitive on trees when $k=3$, we modify Step 1 of the strategy used in Theorem [6 for distributing the surplus. Step 2 is unmodified, but for convenience, we give both steps. Let $C=\frac{5}{8}$.

Step 1: Each edge $(v, u) \in E_{+}^{s}$ transfers a value of $\frac{7}{8}$ to its parent vertex, $v$, and a value of $\frac{1}{8}$ to its child vertex, $u$. Each edge $(v, u) \in E_{+}^{d}$ transfers its surplus of $1-C=\frac{3}{8}$ to its parent vertex, $v$.

For each vertex $v$, let $m(v)$ denote the value transferred to $v$ in this step.

Step 2: Consider in turn all vertices $v \in V$.

(a) If the vertex $v$ has a parent edge $e^{\prime} \in E_{-}$, then $v$ transfers a value of $\min \{m(v), C\}$ to $e^{\prime}$.

(b) Any value remaining at $v$ is distributed equally among the child edges of $v$ belonging to $E_{-}$.

For each edge $e$, let $m_{v}(e)$ denote the value transferred from $v$ to $e$ in this step.

This finishes the description of the strategy.

Fix an edge $e=(x, y) \in E_{-}$. We need to show that $m_{x}(e)+m_{y}(e) \geq C=$ $\frac{5}{8}$. First, note that if $d_{+}^{s}(y) \geq 1$, then $m(y) \geq \frac{7}{8}$ and we are done. Also, if $d_{+}^{d}(y) \geq 2$, then $m(y) \geq 2(1-C)=\frac{6}{8}$, and again we are done. Thus, we may assume that $d_{+}^{s}(y)=0$ and $d_{+}^{d}(y) \leq 1$. We now show that $e$ receives a value of at least $\frac{5}{8}$ in all such cases.

Case 1: $d_{+}(y)=1$. In this case, $m_{y}(e)=\frac{3}{8}$, so we just need to show that $m_{x}(e) \geq \frac{2}{8}$. Note that $d_{+}(x) \geq k-d_{+}(y)=2$. Thus, $x$ has at least one child edge belonging to $E_{+}$.

- Case 1.1: The parent edge of $x$ belongs to $E_{+}^{s}$. In this case, the parent edge of $x$ contributes a value of $\frac{1}{8}$ to $m(x)$.

- Case 1.1.1: $d_{-}(x)=3$. In this case, $d_{+}^{s}(x)=d_{+}(x) \geq 2$, and therefore at least one child edge of $x$ belongs to $E_{+}^{s}$. It follows that $m_{x}(e) \geq$ $\frac{1}{3}\left(\frac{1}{8}+\frac{7}{8}\right)=\frac{1}{3}>\frac{2}{8}$.

- Case 1.1.2: $d_{-}(x) \leq 2$. Since $x$ has at least one child edge in $E_{+}$, $m_{x}(e) \geq \frac{1}{2}\left(\frac{1}{8}+\frac{3}{8}\right)=\frac{2}{8}$. 
- Case 1.2: The parent edge of $x$ belongs to $E_{+}^{d}$. Since in this case, $d_{+}^{d}(x) \geq 1$, it follows that $d_{-}(x) \leq 2$. Thus, we have only the following two subcases:

- Case 1.2.1: $d_{-}(x)=2$. At least one child edge of $x$ must belong to $E_{+}^{s}$. Thus, $m_{x}(e) \geq \frac{1}{2} \cdot \frac{7}{8}>\frac{3}{8}$.

- Case 1.2.2: $d_{-}(x)=1$. Since at least one child edge of $x$ belongs to $E_{+}$, $m_{x}(e) \geq \frac{3}{8}$.

- Case 1.3: The parent edge of $x$ belongs to $E_{-}$. In this case, $x$ has at most two child edges belonging two $E_{-}$. Furthermore, $x$ has at least two child edges in $E_{+}$and at least one of them belongs to $E_{+}^{s}$. Thus, $m_{x}(e) \geq \frac{1}{2}\left(\frac{3}{8}+\frac{7}{8}-\frac{5}{8}\right)>\frac{2}{8}$.

- Case 1.4: $x$ has no parent edge. Since $d_{+}(x) \geq 2, m_{x}(e) \geq \frac{1}{3} \cdot 2 \cdot \frac{3}{8}=\frac{2}{8}$.

Case 2: $d_{+}(y)=0$. In this case, $d_{+}(x)=3$, so $x$ has at least two child edges belonging to $E_{+}$. Furthermore, $d_{+}^{s}(x) \geq 1$. We show that $m_{x}(e) \geq \frac{5}{8}$ in all subcases.

- Case 2.1: The parent edge of $x$ belongs to $E_{+}^{s}$.

- Case 2.1.1: $d_{-}(x)=3$. In this case, $d_{+}^{s}(x)=3$, and hence $m_{x}(e)=$ $\frac{1}{3}\left(\frac{1}{8}+2 \cdot \frac{7}{8}\right)=\frac{5}{8}$.

- Case 2.1.2: $d_{-}(x)=2$. In this case, $d_{+}^{s}(x) \geq 2$, and hence, $m_{x}(e) \geq$ $\frac{1}{2}\left(\frac{1}{8}+\frac{7}{8}+\frac{3}{8}\right)>\frac{5}{8}$.

- Case 2.1.2: $d_{-}(x)=1$. Two child edges of $x$ must belong to $E_{+}$and so $m_{x}(e) \geq \frac{1}{8}+2 \cdot \frac{3}{8}>\frac{5}{8}$.

- Case 2.2: The parent edge of $x$ belongs to $E_{+}^{d}$. In this case, $d_{-}(x) \leq 2$. Thus, $m_{x}(e) \geq \frac{1}{2}\left(\frac{7}{8}+\frac{3}{8}\right)=\frac{5}{8}$.

- Case 2.3: The parent edge of $x$ belongs to $E_{-}$. In this case, $x$ has at least three child edges in $E_{+}$, and at least two of them belong to $E_{+}^{s}$. Moreover, $x$ has at most two child edges belonging to $E_{-}$. Thus, $m_{x}(e) \geq \frac{1}{2}\left(2 \cdot \frac{7}{8}+\right.$ $\left.\frac{3}{8}-\frac{5}{8}\right)=\frac{6}{8}$.

- Case 2.4: $x$ has no parent edge.

- Case 2.4.1: $d_{-}(x)=3$. In this case, $d_{+}^{s}(x)=3$. Thus, $m_{x}(e)=\frac{1}{3} \cdot 3 \cdot \frac{7}{8}=$ $\frac{7}{8}$.

- Case 2.4.2: $d_{-}(x) \leq 2$. Since $d_{+}^{s}(x) \geq 1, m_{x}(e) \geq \frac{1}{2}\left(\frac{7}{8}+2 \cdot \frac{3}{8}\right)>\frac{6}{8}$.

We now show that the analysis in Theorem 8 is tight by showing that $C_{\mathrm{NF}}^{\mathrm{TREE}}(3)=\frac{5}{8}$. This is done by creating an adversary graph which combines the two cases (cases 1.1.2 and 2.1.1) from the proof of Theorem 8 for which the strategy used for distributing the surplus could only guarantee a value of exactly $\frac{5}{8}$.

Theorem $9 C_{\mathrm{NF}}^{\mathrm{TREE}}(3)=\frac{5}{8}$.

Proof The lower bound follows from Theorem 8 . For the upper bound, let $N$ be an integer divisible by 3 . The adversary graph for $N=3$ is illustrated in Fig. 2, The adversary first reveals $2 N$ isolated edges (shown as the top vertical edges in Fig. 2). For $1 \leq i \leq 3$, denote by $M_{i}$ the subset of these $2 N$ edges colored with the color $i$ by NEXT-FIT. Then, the adversary reveals a path 


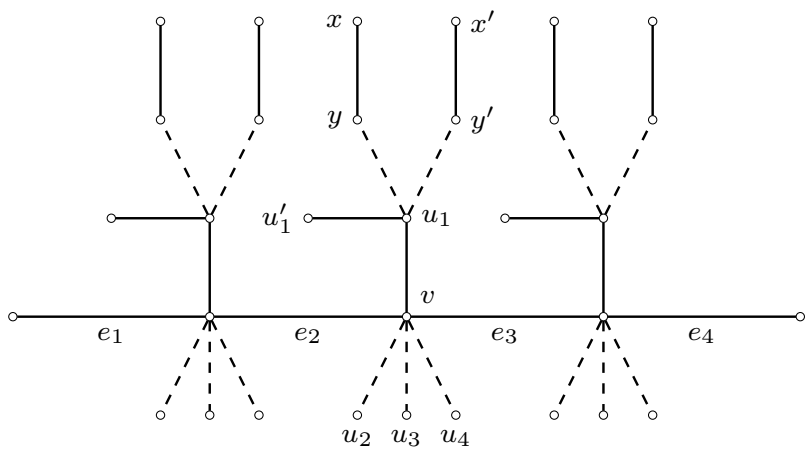

Fig. 2 The adversary graph used in the proof of Theorem 9 when $N=3$. Solid edges are colored by NEXT-Fit and dashed edges are rejected by NEXT-FIT.

$P=\left\langle e_{1}, \ldots, e_{N+1}\right\rangle$ consisting of $N+1$ new edges, revealing the edges from left to right. An inner vertex of $P$ is a vertex of degree 2. For each inner vertex $v$ of $P$, the adversary reveals four edges $\left(v, u_{1}\right), \ldots,\left(v, u_{4}\right)$. Note that $\operatorname{NeXT-Fit~colors~}\left(v, u_{1}\right)$ with the unique color $c \in \mathcal{C}_{1,3} \backslash \mathcal{C}_{v}$ at $v$ and rejects the other three edges. The adversary then reveals an edge $\left(u_{1}, u_{1}^{\prime}\right)$ which is colored with the color $(c+1) \bmod 3$ by NEXT-FIT. Finally, the adversary picks two distinct isolated edges $(x, y),\left(x^{\prime}, y^{\prime}\right) \in M_{(c+2) \bmod 3}$ and reveals two new edges $\left(u_{1}, y\right)$ and $\left(u_{1}, y^{\prime}\right)$. NEXT-FIT rejects both of these edges. The adversary continues with the next inner vertex (unless $v$ was the last inner vertex) and repeats the above procedure. Note that for each $c \in \mathcal{C}_{1,3}$, there are $N / 3$ inner vertices on the path for which $\mathcal{C}_{1,3} \backslash \mathcal{C}_{v}=\{c\}$. Thus, the adversary does not run out of edges to pick from $M_{c}$ (in fact, it uses all $\frac{2}{3} N$ of them). This finishes the description of the adversary strategy.

NEXT-Fit colors the $2 N$ isolated edges, the $N+1$ edges of the path $P$, and for each inner vertex $v$ it colors $\left(v, u_{1}\right)$ and $\left(u_{1}, u_{1}^{\prime}\right)$. Thus, $\operatorname{NF}(I)=$ $2 N+(N+1)+2 N=5 N+1$. On the other hand, Opt rejects all edges of the path $P$. Furthermore, for each inner vertex $v$, Opt rejects $\left(v, u_{1}\right)$. The remaining edges form a graph with maximum degree 3 and hence, Opt can color all of these $8 N$ edges. It follows that $\mathrm{NF}(I)=\frac{5}{8} \mathrm{OPT}(I)+1$. This shows that NEXT-FIT cannot be better than $\frac{5}{8}$-competitive.

\section{Open Problems}

Finding optimal online algorithms for EDGE- $k$-COLORING in general and on other classes of graphs is an interesting open problem. We believe that the techniques used in the proofs of Theorems 4 and 6 can be generalized to, e.g., graphs of bounded degeneracy. In particular, graphs of bounded degeneracy can be oriented so that each vertex has bounded outdegree and the resulting digraph is acyclic. This makes it possible to use strategies for redistributing the surplus similar to the ones we have used for trees. 
Deciding whether there is an algorithm better than FIRST-FiT on trees would also be interesting. Such an algorithm could only be significantly better for small values of $k$, and it would have to be both randomized and unfair.

Acknowledgment. The authors would like to thank the anonymous reviewers for helpful comments on this work and its presentation.

\section{References}

1. Eric Bach, Joan Boyar, Leah Epstein, Lene M. Favrholdt, Tao Jiang, Kim S. Larsen, Guo-Hui Lin, and Rob van Stee. Tight bounds on the competitive ratio on accommodating sequences for the seat reservation problem. Journal of Scheduling, 6(2):131-147, 2003.

2. Amotz Bar-Noy, Rajeev Motwani, and Joseph (Seffi) Naor. The greedy algorithm is optimal for on-line edge coloring. Information Processing Letters, 44(5):251-253, 1992.

3. Allan Borodin and Ran El-Yaniv. Online Computation and Competitive Analysis. Cambridge University Press, 1998.

4. Joan Boyar and Lene M. Favrholdt. The relative worst order ratio for online algorithms. ACM Transactions on Algorithms, 3(2):22, 2007.

5. Joan Boyar, Lene M. Favrholdt, and Kim S. Larsen. The relative worst-order ratio applied to paging. Journal of Computer and System Sciences, 73:818-843, 2007.

6. Joan Boyar and Kim S. Larsen. The seat reservation problem. Algorithmica, 25(4):403417, 1999.

7. Zhi-Zhong Chen, Sayuri Konno, and Yuki Matsushita. Approximating maximum edge 2-coloring in simple graphs. Discrete Applied Mathematics, 158(17):1894-1901, 2010.

8. Martin R. Ehmsen, Lene M. Favrholdt, Jens S. Kohrt, and Rodica Mihai. Comparing first-fit and next-fit for online edge coloring. Theoretical Computer Science, 411(1618):1734-1741, 2010

9. Lene M. Favrholdt and Morten Nyhave Nielsen. On-line edge-coloring with a fixed number of colors. Algorithmica, 35(2):176-191, 2003.

10. Uriel Feige, Eran Ofek, and Udi Wieder. Approximating maximum edge coloring in multigraphs. In Proceedings of the 5th International Workshop on Approximation Algorithms for Combinatorial Optimization, volume 2462 of LNCS, pages 108-121, 2002.

11. Marcin Kamiński and Łukasz Kowalik. Beyond the vizing's bound for at most seven colors. SIAM Journal of Discrete Mathematics, 28(3):1334-1362, 2014.

12. Anna R. Karlin, Mark S. Manasse, Larry Rudolph, and Daniel Dominic Sleator. Competitive snoopy caching. Algorithmica, 3:77-119, 1988.

13. Hal A Kierstead. Coloring graphs on-line. In Online Algorithms, pages 281-305. Springer, 1998.

14. Adrian Kosowski. Approximating the maximum 2- and 3-edge-colorable subgraph problems. Discrete Applied Mathematics, 157(17):3593 - 3600, 2009.

15. Romeo Rizzi. Approximating the maximum 3-edge-colorable subgraph problem. Discrete Mathematics, 309(12):4166 - 4170, 2009.

16. Daniel D. Sleator and Robert E. Tarjan. Amortized efficiency of list update and paging rules. Communications of the ACM, 28(2):202-208, 1985

17. Andrew Chi-Chih Yao. Probabilistic computations: Toward a unified measure of complexity (extended abstract). In Proceedings of the 18th Annual Symposium on Foundations of Computer Science, pages 222-227, 1977. 